\title{
MADA: a web service for analysing DNA methylation array data
}

\author{
Xinyu Hu${ }^{1}$, Li Tang ${ }^{1}$, Linconghua Wang ${ }^{1}$, Fang-Xiang $\mathrm{Wu}^{2}$ and Min $\mathrm{Li}^{1 *}$ (D) \\ From 15th International Symposium on Bioinformatics Research and Applications (ISBRA'19) \\ Barcelona, Spain. 3-6 June 2019
}

\footnotetext{
* Correspondence: limin@csu.edu.cn ${ }^{1}$ School of Computer Science and Engineering, Central South University, Changsha, China Full list of author information is available at the end of the article
}

\begin{abstract}
Background: DNA methylation in the human genome is acknowledged to be widely associated with biological processes and complex diseases. The Illumina Infinium methylation arrays have been approved as one of the most efficient and universal technologies to investigate the whole genome changes of methylation patterns. As methylation arrays may still be the dominant method for detecting methylation in the anticipated future, it is crucial to develop a reliable workflow to analysis methylation array data.

Results: In this study, we develop a web service MADA for the whole process of methylation arrays data analysis, which includes the steps of a comprehensive differential methylation analysis pipeline: pre-processing (data loading, quality control, data filtering, and normalization), batch effect correction, differential methylation analysis, and downstream analysis. In addition, we provide the visualization of pre-processing, differentially methylated probes or regions, gene ontology, pathway and cluster analysis results. Moreover, a customization function for users to define their own workflow is also provided in MADA.

Conclusions: With the analysis of two case studies, we have shown that MADA can complete the whole procedure of methylation array data analysis. MADA provides a graphical user interface and enables users with no computational skills and limited bioinformatics background to carry on complicated methylation array data analysis. The web server is available at: http://120.24.94.89:8080/MADA
\end{abstract}

Keywords: DNA methylation, Normalization, Differential methylation analysis, Downstream analysis 


\section{Background}

DNA methylation of cytosine residues at CpG dinucleotides is one of the most extensive studied epigenetic modifications due to its role in biological processes and complex diseases [1]. Developments in high-throughput evaluation of DNA methylation adopting the second-generation sequencing or microarrays technologies have made it possible to quantify DNA methylation of CpG sites in the whole human genome. The prospect of DNA methylation in improving our understanding of disease mechanism such as tumour detection [2] and classification [3] combined with the current trend of expanding coverage and decreasing cost of DNA methylation microarrays has led to the explosive growth of these technologies. Illumina Infinium Human Methylation450 BeadChip (450 k) array is one of the most cost-effective ways to analyse DNA methylation in the human genome, which targets $96 \%$ of CpG islands and covers more than 450,000 CpG sites [4]. It has been widely used in many large projects [5], such as The International Cancer Genome Consortium (ICGC) and The Cancer Genome Atlas (TCGA) Project [6]. Recently, a reliable genomic approach Methylation EPIC array to study DNA methylation levels and patterns in the whole human genome has developed by Illumina, which covers more than $850,000 \mathrm{CpG}$ sites. As methylation arrays may still maintain its popularity for detecting methylation in the predictable future, so it is significant to provide comprehensive workflow for DNA methylation array analysis.

In recent years, a lot of tools have been proposed to analyse methylation array data with the availability of public data resources. However, most of the tools provided to users are based on command line tools published on Bioconductor. The most popular command line-based platform is Minfi [7], which provides a flexible analysis for Infinium DNA methylation microarrays. However, the complex usage of Minfi causes a significant challenge to the researchers without proficient programming skills. The Bioconductor package ChAMP [8] also has the similar problems. Many biological web platforms $[9,10]$ provided convenient input and output for biologists. Only a few tools are based on methylation array data, such as ADMIRE [11] integrates several normalization methods and provides a statistical method for differentially methylated regions (DMRs), which can only handle $450 \mathrm{k}$ data.. Furthermore, most of these web-based tools can neither cover the whole process of methylation array data analysis, nor provide downstream analyses and result visualization.

Here, we develop a web-based tool MADA which provides a comprehensive analysis of methylation arrays data by integrating quality control, filtering, nine normalization methods, five differential methylation analysis methods and two downstream analysis methods. MADA has good usability and practicability can not only handle the classic methylation array data of $450 \mathrm{k}$, but also analyse the emerging EPIC data. MADA places great emphasis on workflow, which helps finishing the whole analysis through several easy steps. In addition, MADA provides visualization for the entire process, including the results of pre-processing, differentially methylated probes (DMPs) or regions, gene ontology and pathway analysis.

\section{Implementation}

User-friendly graphical user interface

MADA provides a graphical user interface and enables users with no computational skills and limited bioinformatics background to carry on the analysis of sophisticated 
methylation array data. Figure 1 shows the pipeline of MADA. The web server allows users to define workflow on their own, and to complete their analysis through several easy steps. First, users upload custom methylation array datasets including the raw iDAT files and a sample sheet file which describes important sample information in detail. Second, users can adjust a wide range of analysis parameters, such as quality control filtering based on detection $p$ values, the aim of filtering is to remove probes on the sex chromosome, remove probes with SNPs or exclude cross reactive probes, nine normalization methods, batch effect correction, three DMPs detecting methods, four DMRs detecting methods, and visual diagram is provided for each step. Furthermore, pre-processed methylation beta values or $\mathrm{M}$ values matrix files can be uploaded directly to detect DMPs or DMRs. If users are interested in the downstream analysis of DMPs, $\mathrm{GO}$ analysis, pathway analysis and cluster analysis can be used to explore biological significance. After submitting the task, users will receive a notification about the job status and a URL of analysis result, on which all analysis results are generated in the web server and can be downloaded as a compressed archive from the MADA platform.

\section{Data pre-processing and batch effect correction}

The step of data pre-processing is necessary in the overall data analysis process. First, MADA has implemented quality control for raw data which calculates detection $p$-values for each CpG in each sample using the Minfi package [7]. Low quality samples and probes can be easily removed from the analysis by setting the detection p-value in advance. Second, MADA integrates nine typical normalization methods, including BMIQ [12], PBC [13], SWAN [14], Funnorm [15], Illumina [7], Noob [16], SQN [17], Dasen [18], and Raw to decrease the technical variation within and between arrays. Beta Mixture Quantile normalization (BMIQ) is a flexible approach by quantile normalization to fit the distribution of beta-values of Infinium II to the corresponding distribution of beta-values of

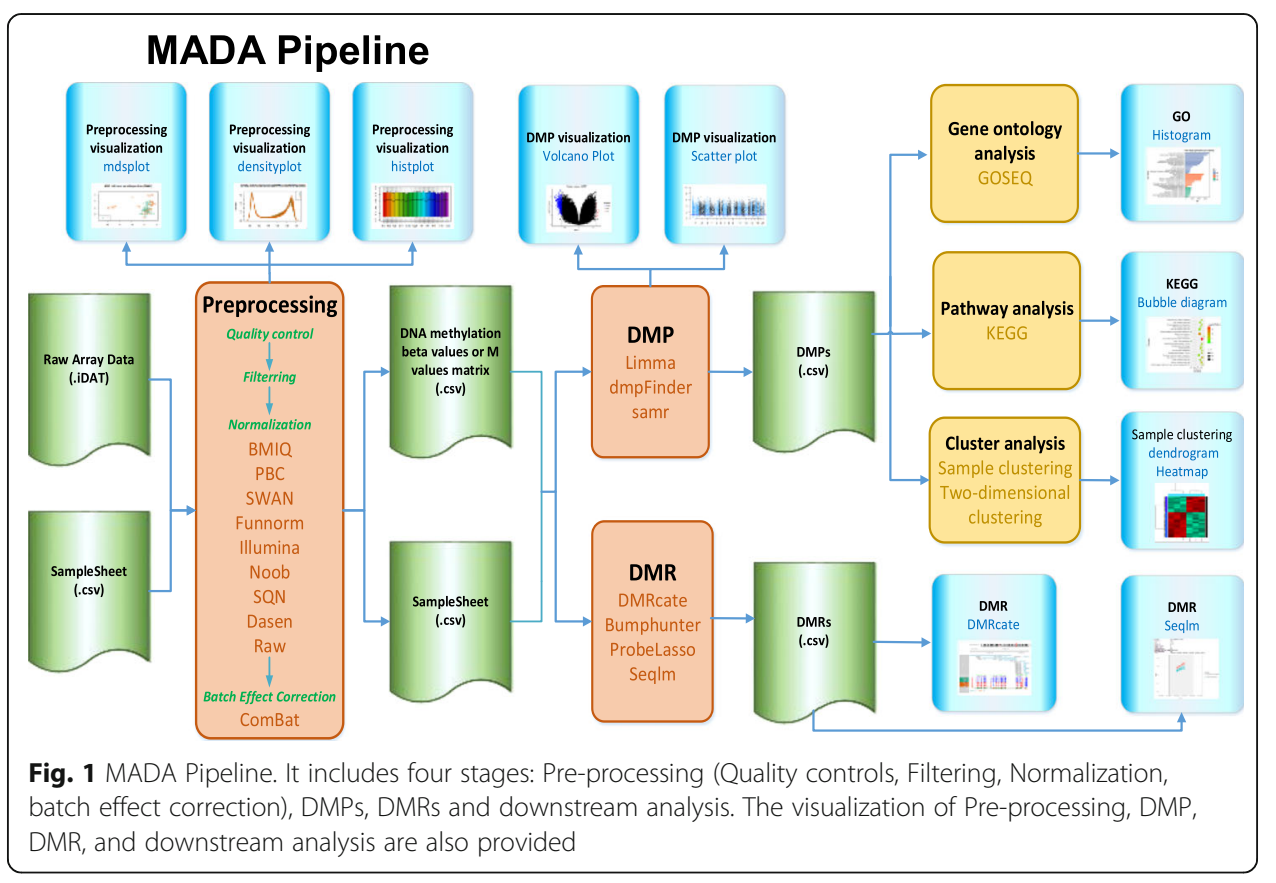


Infinium I, which seems more suitable to be used to normalize data than other methods. Peak-based correction (PBC) method is sensitive to the shape of beta-value density curves which rescales the methylation values of Infinium II to the same modes for methylation values distribution of Infinium I. Subset-quantile Within Array Normalization (SWAN) is a within-array normalization method by altering Infinium I probe data to increases the technical variation. Functional normalization (Funnorm) extends the quantile normalization method to removes unwanted technical variation using control probes. Illumina is not only a within-array normalization but also a between-array normalization method performed pre-processing as Genome Studio. Noob is a novel approach to background correction for Infinium Human Methylation data to account for technical variation in background fluorescence signal. Subset Quantile Normalization (SQN) assumes that the beta values of $\mathrm{CpGs}$ from the same biological category should obey the same density distribution. Dasen is a data driven analysis method to preprocess $450 \mathrm{~K}$ methylation array data. Table S1 of Supplementary Materials 1 lists the normalization tools and corresponding performance. Moreover, MADA provides the function of filtering to exclude probes on the sex chromosomes and probes with SNPs [7] at CpG site, and exclude cross reactive probes [19] in the step of pre-processing.

Due to some potential biological and environmental variables that cannot be measured and may have a significant effect on the measurements of high-throughput biological experiments. MADA implements the batch effect correction with ComBat method from the sva package [20] which was used to further remove technical bias introduced by interrogating samples on the methylation array in different batches. The ComBat function uses an empirical Bayesian framework to adjust to known batches. Eliminating batch effects in differential analysis have been shown highly effective in reducing dependence and stabilizing error rate estimates.

\section{Differential methylation analysis}

After pre-processing, the main purpose of many methylation researches is detecting differentially methylated probe (DMPs) or differentially methylated regions (DMRs) in the human genome. Differential methylation analysis is the most important step, which takes the output file of pre-processed results by different methods as input. Seven popular differential methylation analysis methods (Limma [21],dmrFinder [7], samr [22], DMRcate [23], Bumphunter [24], ProbeLasso [25], seqlm [26]) are integrated in MADA, which based on different statistical methods as shown in Table S2 of Supplementary Material 1. Three of them are used to detect DMPs, Limma is a R package for analysing gene expression and methylation data from microarray technologies and the linear model is considered as the most popular and widely accepted tools for analysing designed experiments and the assessment of differential expression. DmpFinder is a function of $\mathrm{R}$ package Minfi which tests each genomic position for the association between methylation and phenotype. Samr is a package for analysing microarrays data, which correlates many features with an outcome variable, such as a quantitative variable, group indicator or survival time. The remaining four were used to detect DMRs. DMRcate is a novel tool based on a combination of DM-signal smoothing and subsequent threshold specification to agglomerate genomically localized individual DNA methylation CPG sites into discrete DMRs. Bumphunter is a flexible approach for identifying DMRs of biological interest based on 
quantitative high-throughput methods. ProbeLasso is a novel approach to rope in DMRs with DNA methylation array data. Seqlm is an MDL based method for DMR identification by using linear mixed models.

\section{Downstream analysis and visualization}

Go analysis, pathway analysis and cluster analysis are implemented in MADA, which help users to conduct further analysis and discover significant results behind these data. In order to highlight biological processes and reduce complexity, Go analysis is widely used in genome-wide expression studies. GOseq [27] is a software that includes functions to calculate the significance for each Go category amongst differentially expressed genes. MissMethyl [28] provides a KEGG pathway analysis using the gometh function. $\mathrm{R}$ basic clustering function is used in cluster analysis. Downstream analysis uses the output result of DMPs as input, which contains a long list of important CpG sites. Moreover, MADA provides the visualization of pre-processing, DMPs or DMRs, gene ontology and pathway analysis results making use of $\mathrm{R}$, and all visual images can be downloaded on a web page. These tools are listed in Table S3 of Supplementary Material 1.

\section{Results}

Case study of $450 \mathrm{~K}$ data: DNA methylation changes in endometrium and correlation with gene expression during the transition from pre-receptive to receptive phase

This case study describes the approach reported in Scientific Reports [29]. We repeated the parts of pre-processing and differential methylation analysis in their Methylation array data analysis. In this study, the relationship between DNA methylation and gene expression in endometrial biopsies of 17 normal women of childbearing age was studied by genome-wide technique. Seventeen healthy women of childbearing age received pre-receptive and receptive periods in one menstrual cycle. Here we used the "WorkFlow" module to repeat this analysis process. Firstly, Methylation dataset was downloaded from Gene Expression Omnibus (GEO) [30] under accession number of GSE90060. We can get the raw iDAT data and a samplesheet.csv (see 'Help/Contact' in website). On the page of "WorkFlow", step by step we selected "M-value" to conduct differential methylation analyses, and input the detection $p$-value 0.01 as a threshold to filter out unreliable probes, and then chose "TRUE" to remove all probes from X and Y chromosomes affected by SNPs. The pre-processing box contains 9 normalization tools integrated in MADA, here we chose Illumina with the default parameters. The differential methylation analysis box contains 7 differential detection tools, we chose Limma with the default setting, and chose the continuous covariates of age for the design matrix, microarray type is " $450 \mathrm{~K}$ ". The last step, after clicking the "Execute" button, the workflow starts. The definition of the workflow is shown in Fig. 2a. When the operation is finished, the web jumps to the page of "RecentJob" and users can download the final results to their local computer in zip file. In order to compare these results more obviously, we set the adjusted $p$-value as 0.05 to get the same 21,117 significant CpG sites in paper results [29]. The significant CpG sites associated with over 5000 differentially expressed genes between pre-receptive and receptive endometrium as show in Supplementary Material 2. 


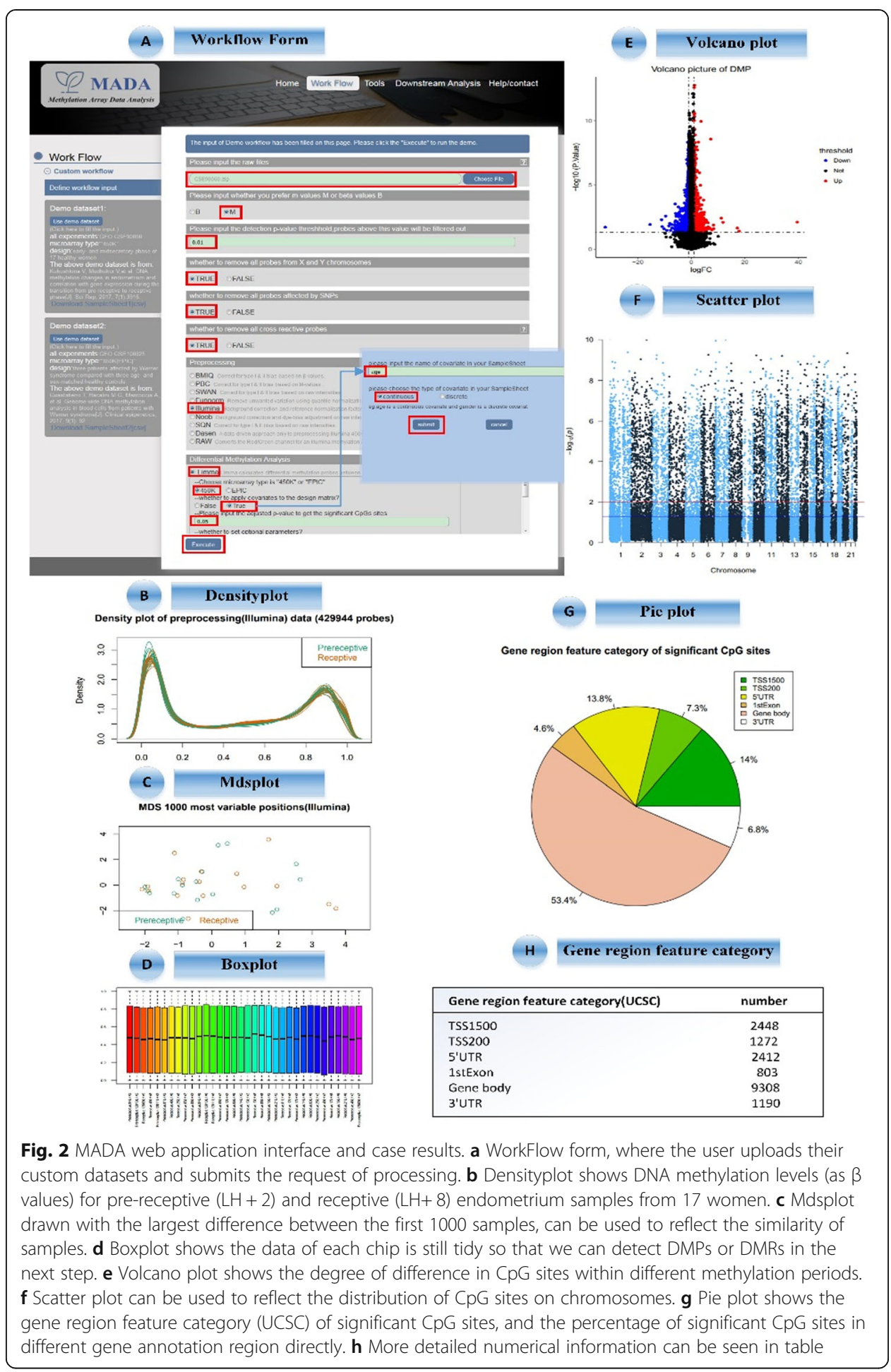

The visualization of pre-processing is as shown in Fig. 2c-d. Densityplot shows DNA methylation levels (as $\beta$ values) for pre-receptive $(\mathrm{LH}+2)$ and receptive $(\mathrm{LH}+8)$ endometrium samples from 17 women. Mdsplot drawn with the largest difference between the first 1000 samples, which can be used to reflect the similarity of samples. From 
boxplot we can see, the data of each chip is still tidy so that we can detect DMPs or DMRs in the next step. The visualization of DMP is shown in Fig. 2e-g. Volcano plot shows the degree of difference in $\mathrm{CpG}$ sites within different methylation periods. Scatter plot can be used to reflect the distribution of $\mathrm{CpG}$ sites on chromosomes. From pie plot we can see the gene region feature category (UCSC) of significant CpG sites, and the percentage of significant CpG sites in different gene annotation region directly. More detailed numerical information can be seen in Fig. $2 \mathrm{~h}$. Sometimes a $\mathrm{CpG}$ site can correspond to multiple genes so that may repeatedly correspond to multiple gene regions. All significant CpG sites information are shown in Supplementary Material 2.

\section{Case study of EPIC data: genome-wide DNA methylation analysis in blood cells from patients with Werner syndrome}

The second case form Clinical Epigenetics [31] uses the Infinium Human Methylation $850 \mathrm{~K}$ BeadChip arrays to verify whether epigenetic changes are associated with Werner syndrome phenotype. As the operation of the workflow mentioned above, step by step we selected beta value to conduct differential methylation analyses, and input the detection $p$-value 0.01 as a threshold to filter out unreliable probes, and chose "TRUE" to remove all probes from $\mathrm{X}$ and $\mathrm{Y}$ chromosomes. Then we chose Funnorm function with the default parameters in pre-processing box. In differential methylation analysis box, we chose Limma with the default setting, chose "False" to apply covariates to the design matrix and chose microarray type as "EPIC". When the operation is finished, the web jumps to the page of "RecentJob" and users can download the final results to their local computer in a zip file, which contains files as same as case study 1 . To verify the effectiveness and reliability of the platform, we list the same results of the top 20 DMPs as the case from Guastafierro's paper which is shown in Table 1.

\section{Comparison of MADA with other typical platforms}

Table 2 shows a comprehensive comparison between MADA and several other typical platforms for Methylation array data analysis including Minfi, ChAMP, watermelon, RnBeads, ADMIRE, Methylumi, missMethyl. Table 2 also lists the systems that the platforms rely on, the installation requirement, the interface, and the functions. As shown in Table 2, MADA has a user-friendly interface, and provides visualization for the entire process, compared with command line-based platforms. In addition, MADA has no requirement for the operating environment, compared with GUI-based platforms. Moreover, MADA integrates more complete methylation array data analysis procedures, and allows users to define their own workflow, compared with other webserver based platforms.

\section{Conclusion}

With the widely use of methylation array data in exploring the associations between DNA methylation and complex diseases, more efficient tools are in urgent need to process HM450K and EPIC array data. In this study we propose a comprehensive pipeline for Illumina methylation array data analysis. The major contributions of the 
Table 1 List of the top 20 DMPs

\begin{tabular}{|c|c|c|c|c|c|}
\hline Probe & chr & pos & $\begin{array}{l}\text { Relation_to } \\
\text { _Island }\end{array}$ & $\begin{array}{l}\text { UCSC_RefGene } \\
\text { _Name }\end{array}$ & Islands_Name \\
\hline cg15294279 & 3 & $174,842,010$ & OpenSea & NAALADL2 & \\
\hline cg00597723 & 5 & $158,691,793$ & S_Shore & UBLCP1 & chr5:158690013-158,690,541 \\
\hline $\operatorname{cg} 16995742$ & 2 & $237,992,612$ & N_Shore & COPS8; & chr2:237994004-237,994,876 \\
\hline cg13956086 & 7 & $2,434,521$ & OpenSea & & \\
\hline $\operatorname{cg} 23432430$ & 12 & $125,538,377$ & S_Shelf & & chr12:125534060-125,534,527 \\
\hline cg26845082 & 3 & $13,555,664$ & OpenSea & & \\
\hline cg17779733 & 22 & $49,589,242$ & OpenSea & & \\
\hline cg23928292 & 12 & $21,815,474$ & OpenSea & & \\
\hline cg06052372 & 16 & $83,967,808$ & OpenSea & & \\
\hline $\operatorname{cg} 10360725$ & 8 & $1.44 \mathrm{E}+08$ & OpenSea & & \\
\hline cg13885829 & 1 & $17,482,041$ & OpenSea & & \\
\hline $\operatorname{cg} 14782559$ & 6 & $33,131,893$ & S_Shelf & COL11A2; & chr6:33129291-33,129,718 \\
\hline cg26822175 & 22 & $27,018,010$ & OpenSea & CRYBA4 & \\
\hline cg20757478 & 6 & $31,012,262$ & OpenSea & & \\
\hline cg18673341 & 7 & $22,481,962$ & OpenSea & MGC87042; & \\
\hline cg17628377 & 5 & $180,121,337$ & OpenSea & & \\
\hline cg08161337 & 22 & $45,814,116$ & OpenSea & $\mathrm{RIBC2}$ & \\
\hline cg15865722 & 11 & $68,860,657$ & OpenSea & & \\
\hline cg07584620 & 1 & $2,265,881$ & N_Shore & MORN1 & chr1:2266007-2,266,432 \\
\hline $\operatorname{cg} 22664298$ & 5 & $128,795,827$ & Island & ADAMTS19 & chr5:128795503-128,797,417 \\
\hline
\end{tabular}

present work lie in MADA provides more comprehensive functions, from preprocessing to differential methylation analysis, and the tools used to downstream analysis are also integrated. MADA further provides the visualization of preprocessing, differentially methylated probes or regions, gene ontology, and pathway analysis results. Besides, MADA allows users to define workflow on their own, and to complete their analysis through several easy steps. MADA encourages researchers to design their customized experiment such as trying different preprocessing methods or combining them to get the best results and use existing datasets to reveal vital new information.

With the analysis of two examples, we have shown that MADA has good usability and practicability can not only handle the classic methylation array data of $450 \mathrm{~K}$, but also analyse the emerging EPIC data. The first case can help researchers to understand the molecular mechanisms governing endometrial biology and receptivity, which highlights the need for similar studies in distinct endometrial cell populations. The second shows DNA methylation changes in the peripheral blood from Werner syndrome patients, which can provide a new insight in the pathogenesis of the disease and help researchers to understand a functional correlation of gene expression and methylation status in some cases.

MADA is meant for the whole process of methylation array data analysis. Whereas most of existing analysis tools are based on command-line published on Bioconductor. MADA provides a user-friendly web-based service as well as source 


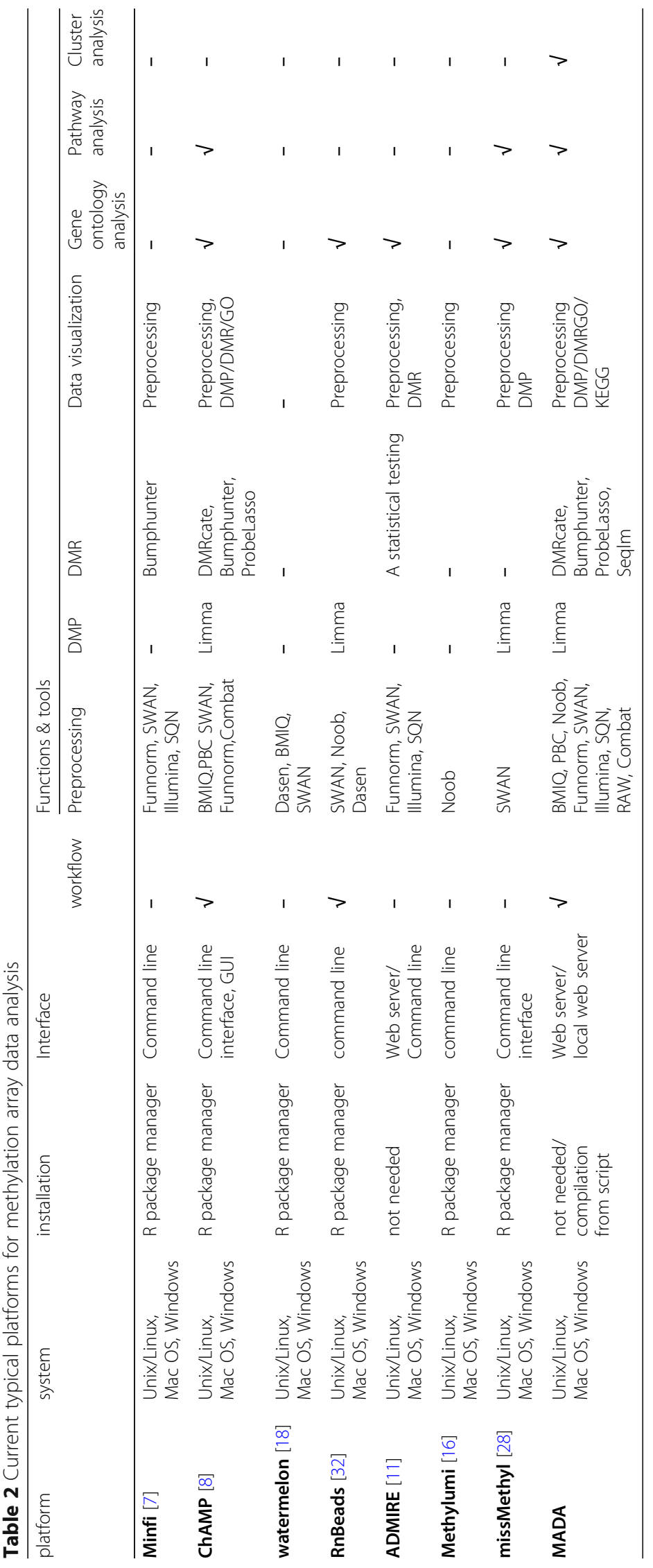


code. However, public web services typically carry out very limited operations on throughput because the workload has to be regulated by the website provider. To solve the above difficulties, MADA will continue to explore advanced strategies and innovative approaches. MADA can potentially provide more smart services such as RNA-seq based conjoint analysis, methylation data based classification of schizophrenia, etc.

\section{Supplementary information}

Supplementary information accompanies this paper at https://doi.org/10.1186/s12859-020-03734-9.

Additional file 1: Supplementary Material 1. This file contains three tables of the list of pre-processing tools, differential methylation analysis tools, differential methylation analysis tools integrated in MADA.

Additional file 2: Supplementary Material 2. This file contains the same significant differentially methylated CpGs from the MADA and the paper of Kukushkina et al.

\section{Abbreviations}

MADA: Methylation arrays data analysis; DMPs: Differentially methylated probes; DMRs: Differentially methylated regions; 450 K: Illumina Infinium Human Methylation450 BeadChip array; EPIC: Illumina Infinium Human Methylation EPIC array

\section{Acknowledgements}

Not applicable.

\section{About this supplement}

This article has been published as part of BMC Bioinformatics Volume 21 Supplement 6, 2020: Selected articles from the 15th International Symposium on Bioinformatics Research and Applications (ISBRA-19): bioinformatics. The full contents of the supplement are available online at https://bmcbioinformatics.biomedcentral.com/articles/supplements/ volume-21-supplement-6.

\section{Authors' contributions}

$\mathrm{XYH}$ carried out the integration studies and coding of methylation array data analysis platform and drafted the manuscript. LT participated in the design of the study and helped to draft the manuscript. LCHW participated in coding of the platform. FXW participated in the design of the study and helped to draft the manuscript. ML carried out the integration studies of methylation array data analysis, participated in the design of the platform and help to draft the manuscript. All authors read and approved the final manuscript.

\section{Funding}

Computational resources were provided by the National Key Research and Development Program of China (No.2019YFA0706200). Study design, data analysis and data interpretation were supported by the National Natural Science Foundation of China U1909208, 61732009, the 111 Project (No. B18059), the Hunan Provincial Science and Technology Program (2019CB1007). Publication fees were covered by the National Key Research and Development Program of China (No.2019YFA0706200).

\section{Availability of data and materials}

The datasets used in case study are available in accession GSE90060 and GSE100825. MADA is an open source collaborative initiative available in the GitHub repository: https://github.com/huxinyu/Methylation. The supplementary materials are provided, and the web server is available at: http://bioinformatics.csu.edu.cn/MADA/Just in case, users can also access MADA at: http://120.24.94.89:8080/MADA.

Ethics approval and consent to participate

Not applicable.

\section{Consent for publication}

Not applicable.

\section{Competing interests}

The authors declare that they have no competing interests.

\section{Author details}

${ }^{1}$ School of Computer Science and Engineering, Central South University, Changsha, China. ${ }^{2}$ Division of Biomedical Engineering and Department of Mechanical Engineering, University of Saskatchewan, Saskatoon SKS7N5A9, Canada. 
Received: 21 May 2020 Accepted: 25 May 2020

Published: 18 November 2020

\section{References}

1. Laird PW. Early detection: the power and the promise of DNA methylation markers. Nat Rev Cancer. 2003;3(4):253.

2. Shen SY, Singhania R, Fehringer G, et al. Sensitive tumour detection and classification using plasma cell-free DNA methylomes. Nature. 2018;563(7732):579.

3. Capper D, Jones DT, Sill M, Hovestadt V, Schrimpf D, Sturm D, et al. DNA methylation-based classification of central nervous system tumours. Nature. 2018;555(7697):469.

4. Teng M, Balch C, Liu Y, Li M, Huang TH, Wang Y, et al. The influence of cis-regulatory elements on dna methylation fidelity. PLoS One. 2012;7(3):32928.

5. Bibikova M, Barnes B, Tsan C, Ho V, Klotzle B, Le JM. High density DNA methylation array with single CpG site resolution. Genomics. 2011:98(4):288-95.

6. Irizarry RA, Ladd-Acosta C, Wen B, Wu Z, Montano C, Onyango P, et al. The human colon cancer methylome shows similar hypo-and hypermethylation at conserved tissue-specific cpg island shores. Nat Genet. 2009:41(2):178-86.

7. Aryee MJ, Jaffe AE, Corrada-Bravo H, Ladd-Acosta C, Feinberg AP, Hansen KD, et al. Minfi: a flexible and comprehensive bioconductor package for the analysis of Infinium DNA methylation microarrays. Bioinformatics. 2014;30(10):1363-9.

8. Morris TJ, Butcher LM, Feber A, Teschendorff AE, Chakravarthy AR, Wojdacz. ChAMP: 450k chip analysis methylation pipeline. Bioinformatics. 2013;30(3):428-30.

9. Li M, Tang L, Wu F-X, Pan Y, Wang J. CSA: a web service for ChIP-Seq analysis. BMC Bioinformatics. 2019. https://doi.org/ 10.1186/s12859-019-3090-0.

10. Zhang J, Zeng M, Kurgan L, Wu F-X, Li M. NetEPD: a network-based essential protein discovery platform. Tsinghua Sci Technol. 2019. https://doi.org/10.26599/TST.2019.9010056

11. Preussner J, Bayer J, Kuenne C, Looso M. ADMIRE: analysis and visualization of differential methylation in genomic regions using the Infinium HumanMethylation450 assay. Epigenetics Chromatin. 2015;8(1):51.

12. Teschendorff AE, Marabita F, Lechner $M$, et al. A beta-mixture quantile normalization method for correcting probe design bias in Illumina Infinium 450 k DNA methylation data. Bioinformatics. 2012;29(2):189-96.

13. Dedeurwaerder S, Defrance M, Calonne E, Denis H, Sotiriou C, Fuks F. Evaluation of the Infinium methylation 450K technology. Epigenomics. 2011;3(6):771-84.

14. Maksimovic J, Gordon L, Oshlack A. SWAN: subset-quantile within array normalization for illumina infinium HumanMethylation450 BeadChips. Genome Biol. 2012;13(6):R44.

15. Fortin JP, Labbe A, Lemire M, Zanke BW, Hudson TJ, Fertig EJ, et al. Functional normalization of 450k methylation array data improves replication in large cancer studies. Genome Biol. 2014;15(11):503.

16. Triche TJ Jr, Weisenberger DJ, Van Den Berg D, Laird PW, Siegmund KD. Low-level processing of Illumina Infinium DNA methylation beadarrays. Nucleic Acids Res. 2013:41(7):e90.

17. Touleimat N, Tost J. Complete pipeline for Infinium human methylation 450K BeadChip data processing using subset quantile normalization for accurate DNA methylation estimation. Epigenomics. 2012;4(3):325-41.

18. Pidsley R, Wong CC, Volta M, Lunnon K, Mill J, Schalkwyk LC. A data-driven approach to preprocessing Illumina 450K methylation array data. BMC Genomics. 2013;14(1):293.

19. Chen YA, Lemire M, Choufani S, Butcher DT, Grafodatskaya D, Zanke BW, et al. Discovery of cross-reactive probes and polymorphic CpGs in the Illumina Infinium HumanMethylation450 microarray. Epigenetics. 2013;8(2):203-9.

20. Leek JT, Storey JD. A general framework for multiple testing dependence. Proc Natl Acad Sci. 2008;105(48):18718-23.

21. Smyth G, Limma K. Linear models for microarray data. Bioinformatics \& Computational Biology Solutions Using R \& bioconductor; 2011. p. 397-420.

22. Tusher VG, Tibshirani R, Chu G. Significance analysis of microarrays applied to the ionizing radiation response [J]. Proc Natl Acad Sci. 2001:98(9):5116-21.

23. Peters TJ, Buckley MJ, Statham AL, Pidsley R, Samaras K, Lord RV, et al. De novo identification of differentially methylated regions in the human genome. Epigenetics Chromatin. 2015;8(1):6.

24. Jaffe $A E$, Murakami $P$, Lee $H$, Leek JT, Fallin MD, Feinberg AP, et al. Bump hunting to identify differentially methylated regions in epigenetic epidemiology studies. Int J Epidemiol. 2012;41(1):200-9.

25. Butcher LM, Beck S. Probe lasso: a novel method to rope in differentially methylated regions with 450K DNA methylation data. Methods. 2015;72:21-8.

26. Kolde R, Märtens K, Lokk K, Laur S, Vilo J. Seglm: an MDL based method for identifying differentially methylated regions in high density methylation array data. Bioinformatics. 2016;32(17):2604-10.

27. Young MD, Wakefield MJ, Smyth GK, Oshlack A. Gene ontology analysis for RNA-seq: accounting for selection bias. Genome Biol. 2010;11(2):R14.

28. Phipson B, Maksimovic J, Oshlack A. missMethyl: R package for analyzing data from Illumina's HumanMethylation450 platform. Bioinformatics. 2015;32(2):286-8.

29. Kukushkina V, Modhukur V, Suhorutšenko M, Peters M, Mägi R, Rahmioglu N, et al. DNA methylation changes in endometrium and correlation with gene expression during the transition from pre-receptive to receptive phase. Sci Rep. 2017;7(1):3916.

30. Barrett T, Troup DB, Wilhite SE, Ledoux P, Evangelista C, Kim IF, et al. Ncbi geo: archive for functional genomics data sets-10 years on. Nucleic Acids Res. 2011;39(Database issue):1005-10.

31. Guastafierro T, Bacalini MG, Marcoccia A, Gentilini D, Pisoni S, Blasio AMD, et al. Genome-wide dna methylation analysis in blood cells from patients with werner syndrome. Clin Epigenetics. 2017;9(1):92.

32. Assenov Y, Müller F, Lutsik P, Walter J, Lengauer T, Bock C. Comprehensive analysis of DNA methylation data with RnBeads. Nat Methods. 2014;11(11):1138.

\section{Publisher's Note}

Springer Nature remains neutral with regard to jurisdictional claims in published maps and institutional affiliations. 